\title{
MARCACIÓN EXPLÍCITA DE FOCO ESTRECHO EN ESPAÑOL
}

\section{INTRODUCCIÓN ${ }^{1}$}

En algunas variedades del español de América, específicamente en regiones de Colombia, Venezuela, Panamá, Ecuador y República Dominicana ${ }^{2}$, son de uso frecuente las construcciones de (1). Quiero señalar que a lo largo de este trabajo retomo ejemplos exclusivamente de la variedad del español bogotano ${ }^{3}$ :

${ }^{1}$ Quiero dar las gracias a Rodrigo Gutiérrez Bravo, María Eugenia Vázquez Laslop, Laura Romero Rangel, Sergio Bogard S. y Valeria Velloro por los comentarios que hicieron a una versión preliminar de este trabajo. Igualmente, el reconocimiento para los lectores anónimos que dictaminaron este artículo. Gracias a todos ellos pude mejorar en lo esencial la propuesta que ahora presento. No sobra decir que la responsabilidad de lo que aquí se dice es exclusivamente mía.

${ }^{2}$ Véase Charles Kany, American-Spanish syntax, The University of Chicago Press, London, 1945; Rufino Cuervo, Apuntaciones críticas sobre el lenguaje bogotano, Instituto Caro y Cuervo, Bogotá, 1955; Hugo Albor, "Uso e interpretación de «ser» en construcciones galicadas y en «él necesita es descansar»", BICC, 41 (1986), 3-277; Mercedes Sedano, "Yo vivo es en Caracas: un cambio lingüístico", en Studies in the Caribbean Spanish dialectology, eds. M.R. Hammond \& M.C. Resnick, Georgetown University Press, Washington, DC, 1988, pp. 115-123 y Hendidas y otras construcciones con ser en el habla de Caracas, Universidad Central de Venezuela, Caracas, 1990; JoHN Lipski, El español de América, Cátedra, Madrid, 1992; JoAQuín Montes, Dialectología general e hispanoamericana, Instituto Caro y Cuervo, Bogotá, 1982; Humberto Toscano, El español en el Ecuador, CSIC, Madrid, 1953, y José CAмасно, "In situ focus in Caribbean Spanish: Towards a unified account of focus", Selected Proceedings of the 9th Hispanic Linguistics Symposium, eds. N. Sagarra \& J. Toribio, Somerville, MA, 2006, pp. 13-23.

${ }^{3}$ El corpus utilizado se levantó en el Instituto Caro y Cuervo y corresponde a treinta entrevistas realizadas a quince hombres y a quince mujeres de diferentes profesiones, edades y niveles educativos. Cada una de las entre- 
(1) a. Ese cemento no va a pegar [es [nada]].

b. Tiene que ir [es [a un restaurante]] a almorzar.

c. Ahí estaría [era [aguantando]] hambre.

d. Teníamos [era [que trabajar mucho]].

Estas construcciones generalmente han sido asociadas con las construcciones perifrásticas de relativo o escindidas, en el sentido de que hay una posible reducción de donde, lo que, que, etc. ${ }^{4}$. Para Moreno Cabrera ${ }^{5}$, las construcciones de (1) son perífrasis copulativas, en las que desaparece totalmente la partícula relacionante (pronombre relativo o conjunción subordinante), con lo que se obtiene una perífrasis especificativa.

Dentro de este mismo orden de ideas, Toribio propone, dentro del marco teórico generativista, que las construcciones de (1) son estructuras pseudo-clefts que carecen de un pronombre relativo ${ }^{6}$. No obstante su similitud existen diferencias. Para tal fin presento un contraste de estas construcciones en (2):

(2) a. A quien compré el carro fue a Pedro.

$a$. [A quien $\varnothing]$ compré $[\mathrm{Ec}]^{7} \ldots$

b. Compré el carro fue a Pedro.

b'. [Op ø] Compré $[\mathrm{Ec}] \ldots$

Las construcciones de ( $2 a$ '), pseudo-cleft, y de $\left(2 b^{\prime}\right)$, 'focalizada', poseen un operador y un complementante nulo, solo que en la primera éstos son evidentes y en la segunda no, sin embargo las dos construcciones, $\left(2 a^{\prime}\right)$ y $\left(2 b^{\prime}\right)$, comparten las características de los operadores nulos ${ }^{8}$.

vistas tiene una duración aproximada de 45 minutos. Hasta donde tengo conocimiento, este corpus no se encuentra publicado en ningún formato. Para la elaboración de este trabajo se utilizó una versión capturada en Word, facilitada por el personal que en el año 2000 trabajaba en la ya desaparecida Maestría en Lingüística. Además, se utilizaron ejemplos tomados de noticieros y de personas de la calle.

${ }^{4}$. R. Cuervo, op. cit. y Sedano, op. cit.

${ }^{5}$ Moreno Cabrera, "Las funciones informativas: las perífrasis de relativo y otras construcciones perifrásticas”, en Gramática descriptiva de la lengua española, eds. V. Demonte e I. Bosque, Espasa, Madrid, 1999, pp. 4245-4302.

${ }^{6}$ Jacqueline Toribio, "Proper government in Spanish subject relativization", Probus, 4 (1992), 291-304.

${ }^{7}$ Utilizo estas abreviaturas: Op.: operador nulo; $\varnothing$ : complementante nulo; Ec.: variable.

${ }^{8}$ Para más detalle de esta posición véanse J. TORIBio, art. cit., y DANA Wheeler, "Portuguese pseudo-clefts: Evidence for free relatives", Papers 
Bosque rechaza la propuesta planteada por Toribio y asegura que estas construcciones no son escindidas, sino que ser, más que un verbo copulativo, es el núcleo de una proyección de foco ${ }^{9}$. En este trabajo comparto los argumentos presentados por Bosque y Albor ${ }^{10}$, con respecto al hecho de que las construcciones de (1) no son escindidas. Aquí me dedicaré a mostrar que ser, en las construcciones de (1), funciona como un marcador de foco in situ.

\section{ORACIONES ESCINDIDAS Y CONSTRUCGIONES CON SER FOCALIZADOR}

Generalmente, las construcciones escindidas están formadas por tres partes: la escisión (EC), la cópula $(\mathrm{CO})$ y la relativa libre (RL) ${ }^{11}$, como en (3):

(3) a. El que compró la patineta fue Juan.

b. Ese balón fue el que recibió María.

c. Es a mi a quien le gusta el chocolate.

d. Donde se vive mejor es aquí.

Si cada una de estas construcciones ${ }^{12}$ tuviera su respectivo correlato indiviso, podríamos observar que el constituyente

from the 18th Regional Meeting of Chicago Linguistic Society, The University of Chicago Press, Chicago, 1982, pp. 507-520.

${ }^{9}$ Este tipo de construcciones no es una pseudo-cleft que contenga un operador QU nulo, ya que ser es un marcador focal del constituyente que le precede. La única similitud de estas construcciones con las escindidas (cleft) es que las dos contienen categorías funcionales morfológicas, como tiempo, persona y número, que son ininterpretables (IgNAcio BosQue, "On focus vs. Wh-movement: The case of Caribbean Spanish”, Sophia Linguistica, 44/45, 1999, 1-32).

${ }^{10}$ I. BosQue, art. cit. y H. Albor, art. cit.

${ }^{11}$ En adelante, utilizo las siguientes abreviaturas: =: frontera de clítico; 3: tercera persona del singular; AC: acusativo; AD: adjunto; CO: cópula; EC: escisión; FADV: frase adverbial; FO: frecuencia fundamental; FOC: foco; FP: frase prepositiva; FV: frase verbal; LOC: locativo; MOD: modo; MP: marcador de predicado; MT: marcador terminal; O: oración; OBJ: objeto; OD: objeto directo; OI: objeto indirecto; PAS: pasado; PERF: perfectivo; RL: relativa libre; REL: relativo; SUJ: sujeto; V: verbo.

${ }^{12}$ Estas construcciones han sido denominadas de diferente forma: oraciones seudo-hendidas o, en inglés, pseudo-cleft sentences (FRANCESCO D’Introno, Sintaxis transformacional del español, Cátedra, Madrid, 1979 y Sintaxis generativa del español. Evolución y análisis, Cátedra, Madrid, 2001; 
escindido, en cursiva, puede ser el sujeto, como en $(3 a)$, el objeto directo, como en ( $3 b)$, el objeto indirecto, como en ( $3 c)$ o el complemento circunstancial, como en $(3 d)$. La parte o el constituyente escindido puede aparecer en uno de los siguientes órdenes: RL CO EC, como en ( $3 a)$, EC CO RL, como en $(3 b)$, y CO EC RL, como en ( $3 c)$. Por su parte, si bien en las construcciones con serfocalizador, como en (4), se puede focalizar un constituyente específico, ya sea un adjunto, como en (4a), el sujeto, como en (4b), o el objeto directo, como en (4c), no va a aparecer ningún nexo relativo $y$, en consecuencia, tratar de buscar un correlato escindido por medio de una paráfrasis desvirtúa completamente el sentido de la construcción con focalizador ser:

(4) a. Empezaron fue con la casita prefabricada.

$a$. Fue con la casita prefabricada que empezaron.

b. El bus lo pagaba era mi papá.

$b$. El que/quien (*lo) pagaba el bus era mi papá.

c. Vi fue la parte de la casa.

c'. Lo que vi fue la parte de la casa.

La creación de un correlato escindido implica ante todo una reorganización en el orden de los constituyentes, como en $\left(4 a^{\prime}\right)$, el cual iría en contra de la naturaleza misma de las construcciones focalizadas, ya que en estas construcciones el focalizador ser nunca puede aparecer en posición inicial absoluta; construcciones como * fue con la casita prefabricada empezaron o * era mi papá pagaba el bus no son posibles. De igual forma, en la paráfrasis, tras la búsqueda de la gramaticalidad, se eliminan algunas partículas morfológicas (el clítico de concordancia) de la construcción focalizada, como en ( $4 b^{\prime}$ ), ya que no es posible mantener en la paráfrasis el objeto directo antepuesto al verbo.

M. Sedano, op. cit. y Amparo Morales, "Recursos expresivos, condicionamientos pragmático y variación dialectal: las oraciones hendidas", $S p C$, 2, 2005, 77-92); oraciones pseudo-escindidas (MARÍA Jesús FERNÁndEZ LEBORANS, "Sobre formas de ambigüedad de las oraciones escindidas: sintaxis y discurso", EstL, 2001, núm. 15, 5-52); estructuras ecuacionales (Emilio Alarcos Llorach, Estudios de gramática funcional del español, $3^{\mathrm{a}}$ ed., Gredos, Madrid, 1980), y perífrasis de relativo (Juan Carlos Moreno Cabrera, "Las perífrasis de relativo", Serta Philologica. F. Lázaro Carreter. Natalem diem 60 celebranti dicata, Cátedra, Madrid, 1983, pp. 455-467 y "Las funciones informativas...”). 
Por una parte, en las construcciones con ser focalizador se puede determinar que este marcador tiene alcance sobre un constituyente o un adjunto específico, mientras que en las escindidas sólo se podrá conocer el alcance que tiene la cópula si se recurre a movimientos particulares. Es posible encontrar el focalizador ser distribuido de la siguiente manera: nos tocó fue venderla ocupada o nos tocó venderla fue fiada; en el primer caso el focalizador tiene alcance sobre el verbo principal y en el segundo sobre el adjetivo. Por otra parte, si se opta por la escisión se obtiene: lo que nos tocó fue venderla fiada o fiada fue como nos tocó venderla. En la primera oración, la cópula tiene alcance sobre el verbo principal y el adjetivo, ya que no tiene sentido una construcción como: * lo que nos tocó venderla fue fiada; sin embargo, para obtener la escisión del adjetivo se necesita que fiada vaya en posición prominente y se resalte su naturaleza semántica con el relativo como, por ejemplo en fue fiada como nos tocó venderla.

Un hecho lingüístico por medio del cual se muestra que las construcciones con focalizador sery las escindidas no comparten rasgos es que en estas últimas construcciones puede aparecer el focalizador ser, como en (5):

(5) a. Lo que a él le importa es más que todo es dormir y comer.

$a$ '. Lo que a él le importa es más que todo dormir y comer.

$b$. Es que le da a uno es hasta vergüenza de ver un viejo ahí.

$b$. Es que le da a uno hasta vergüenza de ver un viejo ahí.

Las construcciones de $(5 a)$ y $(5 b)$ son construcciones escindidas integradas por sus tres partes básicas (RL CO EC), y al interior de cada una de ellas aparece, además, el serfocalizando una parte de estas construcciones. Para hacer el contraste entre las escindidas con focalizador ser, presento en $\left(5 a^{\prime}\right)$ y $\left(5 b^{\prime}\right)$ el respectivo correlato escindido. Si las construcciones con focalizador serfueran las mismas escindidas entonces sería imposible que ser apareciera en una de estas construcciones. Esto muestra que el focalizador ser no debe confundirse con la cópula ser y que de la misma manera que aparece en oración indivisas, también puede aparecer en construcciones escindidas.

La similitud que existe entre escindidas y construcción focalizada es la presencia de serque contiene categorías funcionales morfológicas, como tiempo, persona y número, que son inin- 
terpretables ${ }^{13}$. La ininterpretabilidad se basa en que la cópula y el focalizador ser no siempre concuerdan en tiempo, número y persona con la parte o el constituyente que les antecede o sigue. Ante la pregunta: ¿cómo se interpretan morfológicamente los rasgos de este verbo focal?, Bosque señala que el verbo ser, en este tipo de construcciones, más que una categoría léxica es una categoría funcional, ya que se trata de un marcador gramatical con el que se hace la interpretación semántica informativa de un constituyente. No obstante, los rasgos morfológicos verbales flexivos que presenta se deben a que es una categoría natural. De la misma manera, como ya lo había anunciado Bosque, en las construcciones que aquí estudio, los rasgos de número y persona, como en (6), no son interpretables, mientras que el de tiempo, como en (7), excepto $(7 d)$ y $(7 e)$, sí lo es:

(6) a. Tengo que comparar es datos.

$b$. Tengo que comparar son datos.

Generalmente, la flexión de número de ser concuerda con el de la frase nominal que le sigue. Sin embargo, este rasgo presenta variación en el sentido de que puede aparecer con uno u otro, esto es, singular, como en ( $6 a)$, o plural, como en $(6 b)$. Lo mismo ocurre con la flexión de tiempo, como en (7):

(7) a. Pero no cargo ahí navajas, sino cargo es la plata.

b. Nos dieron cien mil pesos, porque nos tocó fue venderla fiada.

c. Ya le digo, se emborrachaban era a punta de chicha.

d. El tráfico estaba es congestionado.

e. Me tocará caminar es quince cuadras.

Como se evidencia en estos casos, en esta marca focalizadora se repite la flexión verbal del verbo principal, como en $(7 a)$, car-

${ }^{13}$ Para I. BosQue (art. cit.), las construcciones escindidas y las del focalizador ser se parecen en que el verbo ser "copia" generalmente la flexión de tiempo del verbo principal, y que este focalizador es similar a los verbos copulativos de las escindidas respecto al hecho de que el tiempo de los verbos copulativos en las escindidas es expletivo, ya que no tiene significado deíctico. Por ejemplo, las construcciones de (I) no son sinónimas, mientras que las de (II) tienen exactamente el mismo significado: (I) a. Aquí ocurre lo que mencionaste; $b$. Aquí ocurrió lo que mencionaste. (II) $a$. Aquí es donde ocurrió lo que mencionaste; $b$. Aquí fue donde ocurrió lo que mencionaste. La flexión de tiempo pasado (expletiva) de ser, como en (II $a$ ), expresa un tiempo que no puede ser interpretado con respecto al tiempo de mencionaste. 
go-presente, es-presente; (7b), tocó-pretérito, fue-pretérito; y $(7 c)$, emborrachaban-copretérito, era-copretérito. Pero en ( $7 d)$, el tiempo del verbo principal estaba-copretérito y (7e), tocará-futuro, no concuerda con el tiempo presente del focalizador ser. Bajo esta perspectiva, ser, más que un verbo copulativo, es un marcador de foco que aún se encuentra en proceso de gramaticalización, ya que no posee la autonomía morfológica propia de un clítico, como por ejemplo la partícula $c h u$ del quechua huallaga del Perú, que marca qué constituyente dentro de una construcción, como en (8), donde está focalizado ${ }^{14}$.

(8) a. María Hwan-ta maqa-sha María Juan-ACC golpear-3SUJ.PERF

'María golpeó a Juan'.

b. María Hwan-ta maqa-sha=chu? María Juan-ACG golpear-3SUJ.PERF=PREGUNTA ‘¿María golpeó a Juan?'.

c. María Hwan-ta=chu maqa-sha?

‘¿Fue a Juan quien golpeó María?’.

En esta lengua, las preguntas de respuesta sí o no están marcadas con el clítico chu, cuya función focalizadora depende del constituyente al que se le une, pues en (8b) este clítico se une al verbo, por lo tanto se focaliza toda la pregunta, mientras que en $(8 b)$ se une al objeto y, en consecuencia, sólo focaliza esta parte de la oración. Como veremos más adelante, el marcador focal ser tiene funciones similares a las del clítico $c h u$, pero su ininterpretabilidad de número, persona y tiempo siguen siendo problemáticas.

\section{TIPOS DE FOCO}

Para argumentar a favor de que ser es un marcador focal in situ retomo el planteamiento de Lambrecht ${ }^{15}$ con respecto a la estructura focal. El foco o foco de la aserción es el componente de una oración estructurada pragmáticamente, en donde la

${ }^{14}$ C.J. Weber, A grammar of Huallaga (Huanuco) Quechua, University of California Press, Berkeley, 1989.

${ }^{15}$ KNUD LAMBRECHT, Information structure and sentence form, Cambridge University Press, Cambridge, 1994, y "An analysis of the merging of S and O in sentence-focus constructions across language”, Studies in Language, 2000, núm. 23, 611-682. 
aserción pragmática (o sea la información que el receptor, después de haber escuchado una emisión, espera conocer, creer o simplemente considera como dada) difiere de la presuposición pragmática (conjunto de proposiciones léxico gramaticales evocadas en una emisión, que el hablante asume que el oyente ya sabe, cree o considera como un hecho en el momento de la enunciación). El denotatum del foco es por definición un elemento de una proposición comunicativamente impredecible ${ }^{16}$. En este orden de ideas, Van Valin y LaPolla ${ }^{17}$ hacen una distinción entre foco y constituyente sintáctico con respecto a dónde puede aparecer éste en la oración, y llaman dominio focal al constituyente sintáctico en el que ocurre el foco. Dicho de otra manera, el medio gramatical que indica el alcance de la aserción en una construcción en contraste con la información presupuesta o la parte topical de la oración es denominada estructura focal ${ }^{18}$.

${ }^{16}$ En una emisión no sólo se comunica la información focalizada sino que este pedazo de información está asociado con el conjunto de suposiciones pragmáticas; por ejemplo, si digo Juan por sí solo no es informativo, pero si digo Juan fue el que te golpeó, o si digo Juan como respuesta a la pregunta, ¿quién te golpeó?, entonces la información focalizada, Juan, completa la proposición abierta, x golpeó al que escucha; esta información corresponde a la presuposición pragmática a partir de la cual crea la aserción de la información, Juan golpeó al oyente; la información focalizada reemplaza a alguien en la presuposición me golpea, pero como se quiere optar por un referente más específico, entonces se utiliza Juan.

${ }^{17}$ Robert Van Valin \& Randy LaPolla, Syntax. Structure, meaning, and function, Cambridge University Press, Cambridge, 1997.

${ }^{18}$ El foco puede recaer sobre cualquier constituyente de la cláusula (R. Van VAlin \& LaPolla, op. cit.). En (I) presento dos casos diferentes: (I) a.-¿Qué le pasó a tu carro? -Mi carro está dañado; b.-¿Qué pasó? -Mi carro está dañado. El dominio focal, o sea el constituyente oracional focalizado, en ( $\mathrm{I} a)$, recae únicamente sobre el predicado, está dañado. En la pregunta, ¿que le pasó a tu carro?, se está evocando el carro del interlocutor; el carro es el tópico sobre el que se debe comentar algo, de tal manera que en la respuesta se focaliza la información del predicado. La estructura de la información de este ejemplo la presento a continuación: oración: mi carro está dañado; presuposición: el carro del interlocutor está disponible como tópico para que se realice un comentario $x$; aserción: $\mathrm{x}$ = está dañado; foco: está dañado; dominio focal: verbo más el predicado. Por otra parte, en (Ib) se focaliza toda la cláusula, mi carro está dañado, pues en la pregunta no se cuestiona sobre algún tipo de información presupuesta, ni tampoco se hace referencia a un tópico, de tal modo que toda la información de la respuesta está focalizada. Veamos ahora la estructura de la información de esta construcción: oración: mi carro está dañado; presuposición: ninguna; aserción: el carro del hablante está dañado; foco: el carro del hablante está dañado; dominio focal: oracional 
Dentro de la taxonomía de los diferentes tipos de estructura del foco, Lambrecht presenta un contraste entre foco estrecho y foco amplio ${ }^{19}$. En el primer caso se focaliza un solo constituyente, como por ejemplo una FN. En el segundo se focaliza más de un constituyente. Dentro de este foco se encuentran el foco predicativo y el foco oracional. En el primer caso se focaliza el predicado, como en mi novia está enferma, y en el segundo toda la oración, como en mi novia está enferma. Véase al respecto la nota 18.

A partir de (9) a (13), presento ejemplos de la estructura del foco estrecho. En este caso, el focalizador ser puede tener dominio sobre el sujeto, el objeto directo, el objeto indirecto, los adjuntos o sus respectivos núcleos. Lambrecht se refiere al 'foco argumental' para lo que formalmente denomina 'foco estrecho', pero nótese que el foco estrecho no está limitado únicamente a los argumentos sino que incluye los adjuntos y los adverbios. En (9), el dominio focal de ser es sobre el sujeto:

(9) a. Sale [es [uno $\left.]_{\mathrm{FOC}}\right]$ con la caneca de la basura.

$b$. En Colombia eso lo maneja [es [Carlos Pérez] ${ }_{\mathrm{FOC}}$ ].

c. Me gusta [es [que una persona sea seria en sus vainas] FOC].

d. Les interesa [es [la vida de ellos $\left.]_{\mathrm{FOC}}\right]_{\text {. }}$

El dominio focal de ser es sobre el sujeto de una construcción intransitiva, como en $(9 a)(9 c)$ y $(9 d)$, o de una transitiva como $(9 b)$. El referente del sujeto puede estar expresado en una oración, como en $(9 c)$, o en una frase nominal, como en $(9 a)$, $(9 b)$ y $(9 d)$.

En (10) el dominio focal de ser es sobre el objeto directo:

(10) a. Yo tengo [es [hambre $\left.]_{\mathrm{FOC}}\right]$.

b. Vi [fue [la parte de la casa $\left.]_{\mathrm{FOC}}\right]$.

c. Vi [fue [que llegó un carro ahí] ${ }_{\mathrm{FOC}}$ ].

d. Le puse [fue [esparadrapo] $\left.{ }_{\mathrm{FOC}}\right]$.

e. Apoyan [es [a otros cursos] $\left.{ }_{\mathrm{FOC}}\right]$.

(argumentos más núcleo). Cualquier constituyente de una cláusula es susceptible a la focalización. Esto quiere decir que potencialmente se puede focalizar un argumento, el verbo y su(s) argumento(s) o una cláusula; como se mostró anteriormente, en ( $\mathrm{I} a$ ), el foco tiene dominio sobre el predicado, y, en $(\mathrm{I} b)$, el dominio focal es oracional. La asociación que existe entre el foco y la estructura de una cláusula se denomina estructura focal.

${ }^{19}$ K. LAmbRecht, op. cit. 
El referente del objeto directo puede estar expresado en una frase nominal simple, como en $(10 a)$, una frase compuesta, como en $(10 b)$, o en una oración, como en $(10 c)$. El objeto puede ser argumento de un verbo biargumental, como en (10d), o puede estar introducido por la preposición $a$, como en $(10 e)$. En (11) el dominio focal de ser es sobre el objeto indirecto:

(11) a. El chocolate me gusta [es [a mí] ${ }_{\text {FOC }}$.

$b$. Le pregunté [fue [al Padre] $\left.{ }_{\text {FOC }}\right]$.

En las dos oraciones de (11), el objeto indirecto sobre el que tiene dominio el focalizador ser está expresado en una frase de dativo.

En (12) el dominio focal de ser es sobre un adjunto:

(12) a. El doctor entró [fue [en la jornada de la mañana] ${ }_{\mathrm{FOC}}$ ].

b. Vayan [es [con él] $\left.{ }_{\mathrm{FOC}}\right]$.

c. Vivía [era [en función de un negocio] FOC].

$d$. Lo iniciamos el acuario [fue [con unas bailarinas $]_{\mathrm{FOC}}$ ].

e. A mi mamá le gusta vivir [es [por allá] ${ }_{\mathrm{FOC}}$ ].

La variedad de adjuntos respecto de su complejidad sintáctica sobre la que tiene dominio el focalizador ser es muy variada, como en (12).

En (13) el dominio focal de ser es sobre el núcleo verbal:

(13) a. Él se [ [FV dejaba [era [gobernar $\left.\left.]_{\mathrm{FOC}}\right]\right]$ de mí.

b. Ahí se la $\left[_{\mathrm{FV}} \text { pasan [es [jugando }\right]_{\mathrm{FOC}}$ ] parqués.

Presento ejemplos que hacen referencia al único dominio que tiene el focalizador ser sobre el núcleo de la perífrasis verbal, como en (13). En el primer caso sobre el infinitivo gobernary en el segundo sobre el gerundio jugando. Un hecho que permite justificar que efectivamente sólo se está focalizando el verbo principal, y no el complemento, es que existe la posibilidad de focalizar el complemento, como en él se dejaba gobernar era de mi y ahi se la pasaban jugando era parqués. De acuerdo con lo planteado por Selkirk ${ }^{20}$, aunque el focalizador aparezca sobre el verbo principal no quiere decir que simplemente se focalice esta parte de la frase verbal, sino que el foco se proyecta a toda la frase

${ }^{20}$ Elisabeth Selkirk, "Sentence prosody: Intonation, stress, and phrasing”, en The handbook of phonology, ed. J. Goldsmith, Blackwell, Oxford, 1995, pp. 550-569. 
verbal. Esta posición no será tomada en cuenta en este estudio, ya que no hay evidencia empírica que justifique este hecho.

Por otra aparte, téngase en cuenta que en contraste con el foco estrecho se encuentra el denominado foco amplio o informativo, que se divide en foco predicativo y foco oracional. El primero generalmente coincide con la forma tradicional a través de la cual se reconoce la información de una oración, esto es tópico y comentario. En este caso, el sujeto es el tópico y el predicado es el comentario o aserción acerca del sujetotópico ${ }^{21}$. En las preguntas de (14), los referentes de las frases nominales esta mujer, el tráfico y la mamá son tópicos que están dentro de la presuposición pragmática, mientras que en sus respectivas respuestas se focalizan sólo las frases que complementan al núcleo del predicado, como de viejita de (14a), congestionado de (14b) y cansona de (14c), y no todo el predicado o el comentario del tópico:

(14) $a . \quad-¿$ De qué murió esta mujer?

-Esta mujer murió [fue [de viejita $]_{\text {FOC }}$.

-*Esta mujer [fue [murió de viejita $\left.{ }_{\mathrm{FOC}}\right]_{\text {. }}$.

b. ¿¿Cómo estaba el tráfico?

-El tráfico estaba [es [congestionado] FOC].

-*El tráfico [es [estaba congestionado] ${ }_{\mathrm{FOC}}$ ].

c. - ¿Cómo está la mamá?

-La mamá se está volviendo [es [cansona] FOC].

-*La mamá [es [se está volviendo cansona] ${ }_{\mathrm{FOC}}$ ].

En estas construcciones, el dominio focal de ser es solamente sobre el complemento del núcleo del predicado, y no son posibles los casos en que el focalizador aparece antes del predicado; por lo tanto, no es posible encontrar focos predicativos. Por su parte, en $(15 a)$, la pregunta no incluye ningún tipo de información que se pueda considerar como tópico. Por tal razón, toda la oración está dentro del dominio focal, como en (15b). La pregunta no evoca formalmente ninguna presuposición pragmática:

(15) a. - ¿Qué pasó?

-Pedro cogió un Germania.

-Pedro cogió [fue [un Germania] ${ }_{\text {FOC }}$.

-*[Fue [Pedro cogió un Germania] FOC]

${ }^{21}$ K. LAmbrecht, op. cit. 
El focalizador ser no puede aparecer en una posición inicial para que tenga dominio sobre toda la oración, como en $(15 d)$; este focalizador sólo tiene dominio focal sobre un constituyente, como en $(15 c)$. Es decir, el dominio focal de ser es sobre un argumento (foco estrecho) o sobre un adjunto. No son posibles el foco predicado (foco amplio) y el foco oracional (foco amplio).

\section{RESTRICCIONES Y FLEXIBILIDAD DEL FOCALIZADOR SER}

Como se dijo más arriba, ser es básicamente un marcador de foco con un dominio estrecho. Su distribución es restringida y tiene dominio sobre cualquier constituyente, sólo en posición posverbal, como en (16) y (17):

(16) a. La buseta la maneja es el hijo del vecino.

b. *Es la buseta la maneja el hijo del vecino.

c. *La buseta la maneja el hijo es del vecino.

d. *El hijo del vecino es maneja la buseta.

(17) a. Yo la pasaba era con él.

b. * Era yo la pasaba con él.

c. *Yo la pasaba con era él.

d. *Yo la era pasaba con él.

El focalizador ser nunca puede aparecer en posición inicial, ya sea que su dominio focal sea un constituyente o toda la oración, como en (16b) y (17b); de igual modo, este focalizador tiene dominio sólo sobre el referente que está expresado en un constituyente, como en (16a) y (17a), pero no es posible en $(16 c)$ y $(17 c)$, ni tampoco en $(16 d)$ y $(17 d)$. El mismo procedimiento se mantiene en las oraciones subordinadas, como en (18):

(18) a. Ellos quieren [es [que les pase uno [es [la plata] $\left.\left.\left.{ }_{\mathrm{FOC}}\right]\right]_{\mathrm{FOC}}\right]$.

b. *Ellos [es [quieren que les pase uno la plata $]_{\mathrm{FOC}}$ ].

c. Es gente que ha trabajado [es [en la sola tierra ${ }_{\mathrm{FOC}}$ ].

d. *[Es parece que ha trabajado en la sola tierra $\left.]_{\mathrm{FOC}}\right]$.

Es posible que el focalizador ser aparezca marcando dos dominios, como en (18a), en donde el primer marcador focal tiene dominio sobre toda la subordinada, ya que esta oración se constituye como objeto directo, y el segundo tiene dominio 
sobre un constituyente interno de la subordinada, la plata, objeto directo del verbo parar. Nótese que tanto en la construcción subordinada de $(18 a)$, como en la relativa (18c), el focalizador sólo tiene dominio sobre el constituyente que se encuentra en posición posverbal.

Dado que el dominio del focalizador ser es estrecho, vale la pena comentar lo que algunos lingüistas han denominado foco estrecho marcado y no marcado. De acuerdo con la perspectiva de Lambrecht; Contreras y Zubizarreta ${ }^{22}$, la diferencia entre este tipo de foco radica en la posición del constituyente focalizado dentro de la oración. Así como en el inglés, en el español el foco estrecho no marcado aparece en la posición final de la oración, como en (19), mientras que el foco estrecho marcado se aleja del extremo derecho, como en (20):

(19) a. Además del ataque de epilepsia le dio $\left[_{\mathrm{OD}}\right.$ [fue [pulmonía] $\left.{ }_{\mathrm{FOC}}\right]$ ].

b. La ambulancia ya llegó $\left[_{\mathrm{FADV}}\left[\right.\right.$ fue $\left.\left.[\text { después }]_{\mathrm{FOC}}\right]\right]$.

c. Yo Fv $\left.\left._{\mathrm{Fv}}[\mathrm{salí} \mathrm{fue} \mathrm{[a} \mathrm{correr}]_{\mathrm{FOC}}\right]\right]$.

d. Se lo dio $\left.\left[_{\mathrm{OI}}[\text { fue [a una compañera }]_{\mathrm{FOC}}\right]\right]$.

(20) a. Me $\left.\left[_{\mathrm{FV}}[\text { gusta es [vivir }]_{\mathrm{FOC}}\right]\right]$ mi vida.

b. Los niños $\left[_{\mathrm{FV}}\left[\operatorname{salen}\left[\mathbf{e s}[\mathrm{a} \text { oír }]_{\mathrm{FOC}}\right]\right]\right.$ solo vulgaridades de ahí de los choferes.

c. Los hijos de uno dicen que [o[es [que uno es anticuado] $\mathrm{FOC}]$ ].

d. El muchacho pelea en las esquinas $\left[_{\mathrm{FP}}[\mathbf{e s}\right.$ [con el revólver en la mano] $\left.{ }_{\mathrm{FOC}}\right]$ ].

La diferencia planteada entre foco estrecho marcado y no marcado no es pertinenete en este estudio, ya que no existe una tendencia que implique un condicionamiento especial para que un constituyente focalizado se ubique en posición final de la construcción, como en (19), ni tampoco para que se ubique en una posición más extrema del lado derecho, como en (20). La ubicación del constituyente focalizado, con respecto a criterios posicionales, sólo está condicionada por el hecho de que debe aparecer en posición posverbal. En términos pragmáticos, el hablante tiene la libertad de marcar, tras propósitos informa-

${ }^{22}$ Loc. cit. y, del mismo Lambrecht, art. cit.; Heles Contreras, A theory of word order with special reference to Spanish, North Holland, Amsterdam, 1976, y María Luisa Zubizarreta, Prosody, focus, and word order, MIT Press, Cambridge, 1998. 
tivos, con ser a cualquier referente que esté expresado en el constituyente que aparezca en posición posverbal.

El focalizador ser se caracteriza porque tiene una estructura rígida, pero esto no restringe su dominio focal, es decir, este focalizador siempre va a tener dominio sobre un constituyente que se encuentre en una determinada posición, lo cual implica que para focalizar los argumentos o los adjuntos de una construcción simple o compuesta que no están dentro del ámbito del focalizador ser, deben desplazarse a una posición adecuada para que se encuentren dentro del alcance del focalizador. Este desplazamiento, que presentan los constituyentes de una construcción para estar dentro del ámbito del focalizador ser, permite establecer una correlación entre estructura focal y sintaxis. En el caso específico de la lengua española, es posible la interacción entre estructura focal rígida del focalizador sery el orden de constituyentes, gracias a la flexibilidad en su ordenamiento, que presenta el orden canónico SVO, en sus posibles configuraciones VSO, OVS y $\mathrm{VOS}^{23}$.

Con respecto al orden de componentes, ha generado mucha polémica el hecho de que el referente que se constituye como sujeto cuando se ubica en una posición inicial presenta algunas restricciones al focalizarse ${ }^{24}$, especialmente cuando se ha establecido que en una construcción transitiva el sujeto ocupa la posición inicial. Sin embargo, este constituyente se desplaza a una posición posverbal para estar dentro del ámbito del focalizador ser, como en (21):

(21) a. Me gusta [es [que una persona sea seria] ${ }_{\text {FOC }}$ ].

b. A ellos les interesa [es [la vida de ellos $\left.{ }_{\mathrm{FOC}}\right]_{\text {. }}$

c. Este año, se hizo [fue [una barca ${ }_{\text {FOC }}$.

d. La buseta la pintan [es [ellos $]_{\text {FOC }}$.

e. El televisor lo dañaron [fueron [los vecinos $\left.{ }_{\mathrm{FOC}}\right]$.

${ }^{23}$ Véase al respecto MARGarita Suñer, Syntax and semantics of Spanish, Georgetown University Press, Washington, DC, 1982; María Luisa Hernanz y José Brucart, La sintaxis (I), Crítica, Barcelona, 1987; Violeta Demonte, Teoría sintáctica: de las estructuras a la relación, Síntesis, Madrid, 1994, y RoDRIGo Gutiérrez Bravo, "La identificación de los tópicos y los focos", $N R F H$, 56 (2008), 363-401, entre otros.

${ }^{24}$ H. Contreras, op. cit., y M.L. Zubizarreta, op. cit. y "Las funciones informativas: tema y foco", en Gramática descriptiva de la lengua española, pp. 4214-4244. 
Obsérvese que en los ejemplos de $(21 a)$ y $(21 b)$ y (21c) no habría desplazamiento de sujeto a una posición posverbal, ya que se considera que, dado el tipo de verbo en los dos primeros casos, y el tipo de construcción en el segundo, la posición canónica de los sujetos es la posverbal. Con estos casos he querido ilustrar simplemente que el ámbito focalizador de ser puede verificarse sobre un sujeto expresado en una oración, como en $(21 a)$, o en una frase nominal, como en $(21 b)$ y $(21 c)$. Por su parte, en el resto de los ejemplos, el sujeto se desplaza a una posición no canónica para ser focalizado. En $(21 d)$, se trata de un sujeto de un verbo transitivo, y en (21e), lo es de un verbo bitransitivo. Antes de continuar, quiero señalar que cuando se focaliza el sujeto de una oración transitiva, como en los dos últimos casos de (21), el objeto directo generalmente se ubica en una posición prominente y aparece un clítico de concordancia; por ahora no dispongo de suficiente evidencia empírica para discutir si la anteposición del objeto directo desempeña algún papel en la manera en como se focaliza el sujeto.

La interacción que se presenta entre la flexibilidad del orden de constituyentes y la rigidez del focalizador ser no es exclusiva de esta variante de la lengua española, sino que este comportamiento es similar al sesotho, lengua que pertenece al grupo sotho de las lenguas bantú del sureste africano. Esta lengua tiene un orden SVO, como en (22), el sujeto debe ser altamente topical, la información que su referente expresa es dada o vieja, y el foco no puede aparecer en posición preverbal ${ }^{25}$ :

$$
\begin{array}{lll}
\text { a. } & \text { Monna o-bed-its-e } & \text { mosimane } \\
& \text { Hombre SUJ-golpear-PERF-MOD } & \text { niño } \\
& \text { 'El/*un hombre golpeó un/al niño'. } & \\
\text { b. } & \text { Mosimane o-bed-its-w-e } & \text { ke monna } \\
\text { niño } & \text { SUJ-golpear-PERF-PAS-MOD por hombre } \\
& \text { 'El/*un niño fue golpeado por un/el hombre'. } \\
\text { c. } & \text { Monna o-fihl il-e } & \\
& \text { hombre SUJ-llegar-PERF-MOD } & \\
& \text { 'El/*un hombre llegó'. }
\end{array}
$$

${ }^{25}$ Catherine Demuth, "Maturation and the acquisition of sesotho passive", Lan, 65 (1989), 56-80 y "Subject, topic and the sesotho passive", JChL, 17 (1990), 67-80; Robert VAN VAlin, "A typology of the interaction of focus structure and syntax", en Typology and the theory of language: From description to explanation, eds. E. Raxilina \& J. Testelec, Languages of Russian Culture, Moscú, 1999, pp. 511-524 y R. VAN VAlin \& R. LaPolla, op. cit. 

d. Ho-ilh-il-e
monna
LOC-llegar-PERF-MOD hombre
'Allí llegó un hombre o un hombre llegó’"26.

La frase nominal preverbal debe ser interpretada como tópico no focalizado, como en $(22 a)$ y (22c), mientras que las frases nominales posverbales pueden ser interpretadas de cualquier manera, dependiendo del contexto, como en (22b). En los verbos intransitivos, el sujeto focalizado aparece después del verbo y la concordancia del sujeto con el verbo es complementada por un marcador locativo de concordancia, como en $(22 d)$.

El impacto de esta restricción puede observarse más claramente en las preguntas QU. Las palabras QU son siempre focales y por eso no pueden aparecer en posición preverbal sino al final de la oración o en posición posverbal en una construcción escindida, como en (23):

En el sesotho no es posible hacer una pregunta donde el pronombre interrogativo aparezca en posición preverbal, como en (23a). En las construcciones pasivas, como en (23b), o en las escindidas, como en $(23 c)$ y $(23 d)$, es posible focalizar el pronombre QU sólo si aparece en posición posverbal. En esta lengua, el dominio focal no tiene alcance sobre toda la oración, y para focalizar el sujeto, que puede estar expresado en una frase nominal o en un pronombre interrogativo, es necesario que éste se encuentre en una posición posverbal, como en las construcciones pasivas de $(22 b)$ y $(23 b)$, en la intransitiva de $(22 d)$, o en una posición poscopular de las construcciones escindidas de $(23 c)$ y $(22 d)$.

\footnotetext{
${ }^{26}$ R. VAN VALIN, art. cit.
} 
Nótese que la estructura focal que despliega el sesotho es similar a la estructura focal de la variedad del español que aquí estudio. En las dos lenguas es imposible focalizar toda la oración, las dos lenguas comparten la restricción o la prohibición de que el constituyente focalizado no debe estar en posición preverbal y se requiere de la flexibilidad que presenta el orden de constituyentes para que se pueda focalizar el sujeto, ya que es imposible focalizarlo en posición inicial.

Teniendo en cuenta la similitud en la estructura focal de la variedad del español bogotano con otras lenguas, el estatus de ser focalizador sería similar al de las partículas que morfológicamente focalizan un constituyente específico. Por ejemplo, el toura, una lengua mande hablada en Costa de Marfil, al Oeste de África, presenta un orden relativamente rígido, SVO, como en (24), y tiene un rico sistema de partículas para marcar el foco:

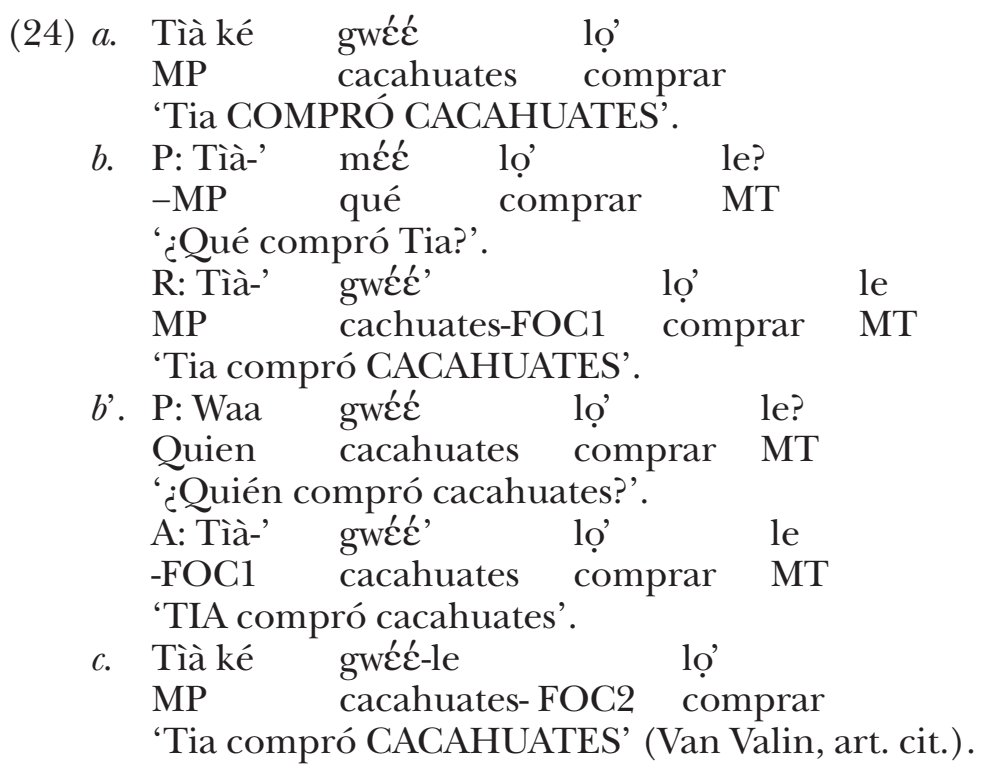

En (24a) se presenta una construcción predicativa básica y focalizada. En (24b) el marcador focal es un clítico tonal (MP) y la construcción de (24c) tiene el mismo orden que (24b), pero está glosada con un marcador terminal (MT). La distinción entre estos dos tipos de foco radica en la presuposición, es decir, en (24b) el foco no es contrastivo y esta marca de foco se emplea en una respuesta que introduce información nueva, mientras que (24c) señala foco contrastivo. Lo interesante, 
para este estudio, de la estructura focal que presenta el toura es que esta lengua ofrece una correlación entre un sistema muy elaborado de marcación de foco flexible y una estructura sintáctica rígida, mientras que en el español bogotano se relacionan una estructura focal rígida y un orden de constituyentes flexible. A pesar de esta gran diferencia, en las dos lenguas se puede focalizar cualquier constituyente o adjunto; incluso en la variante del español bogotano existe la posibilidad de focalizar diferentes constituyentes que forman parte de la misma construcción, como en (25a), o duplicar el focalizador dentro del mismo constituyente, como en (25b):

(25) a. Ellos quieren $\left.\left[{ }_{\mathrm{O}}[\text { es que les pase uno [es [la plata }]_{\mathrm{FOC}}\right]\right]$ FOC] ].

b. Habíamos muchachos que [Fv iban no [era [a estudiar] $\left.\left.\left.\left.\left.{ }_{\mathrm{FOC}}\right]\right]_{\text {sino }}[\text { era [a conseguir }]_{\mathrm{FOC}}\right]\right]\right]_{\text {novia. }}$

En la oración transitiva de (25a) se focaliza tanto el referente de la oración que se constituye como objeto directo subordinado de la oración principal, es que les pase uno es la plata, como el objeto directo de la oración subordinada, es la plata; en (25b), dentro de la construcción relativa se duplica el focalizador en cada uno de los infinitivos coordinados, cada uno por separado, esto es: era a estudiar y era a conseguir. Incluso, la capacidad focalizadora de ser está por encima de los operadores léxicos de foco, como en (26b) y (26c), y también puede focalizar un constituyente de una oración escindida, como en (26f):

(26) a. SolamenteJuan (solamente) compró (solamente) el periódico.

b. Ya la acostumbró fue sólo a alverjas.

c. Como es gente que ha trabajado es únicamente en la tierra.

d. En las juntas de acción comunal siempre debe trabajar es una gente que sean de clase media.

e. Ya se da es cuenta qué tiene uno en la casa.

$f$. Es al vecino al que le robaron fue el carro.

Recuérdese que los focos argumentales pueden aparecer con un adverbio del tipo solamente o únicamente que denotan un sentido de exclusividad, exhaustividad o unicidad como en $(26 a)^{27}$. El sentido expresado por la oración de (26a) depende del alcance

${ }^{27}$ James McCawley, "The focus and scope of only", en Discourse and meaning, eds. B. Partee \& P. Sgall, J. Benjamins, Amsterdam, 1996 y R. Gutí́RREZ BRAVO, art. cit., entre otros. 
que tiene el operador de foco solamente. Al parecer, la capacidad focalizadora de este tipo de adverbios tiene poca relevancia, ya que son susceptibles de ser focalizados por ser. Además, se pueden encontrar casos, como en (26d) y (26e), en donde aparecen adverbios de frecuencia o de puntualidad. De igual manera, el focalizador ser puede aparecer en construcciones escindidas, como en $(26 f)$. No se debe olvidar que estas construcciones se han constituido en un mecanismo de focalización muy común, al menos en el español, el francés, el vasco, el húngaro y algunas lenguas bantú ${ }^{28}$. El hecho de que el focalizador ser coaparezca en construcciones escindidas y en construcciones modificadas por adverbios de diferente naturaleza semántica es muy sugerente sobre la función focalizadora específica de ser. No obstante, se debe tener en cuenta que, translingüísticamente, en las lenguas del mundo se han encontrado diversos mecanismos lingüísticos para señalar el foco, como, por ejemplo, rasgos prosódicos, sintácticos, morfológicos y adverbiales. Estos procedimientos no tienen por qué darse por separado, y las construcciones con focalizador serpuede ser uno de los casos en que se combinan dos o más mecanismos de focalización en una misma oración.

En términos generales, el focalizador sermantiene una estructura focal rígida, pero todos los componentes de la oración pueden ser focalizados si se encuentran en posición posverbal. Esta estructura focal es similar al seshoto porque presenta una restricción de la focalización preverbal, y al toura, porque se pueden focalizar no solamente los argumentos, sino también los adjuntos.

\section{FOCALIZADOR SER Y ENTONACIÓN}

La naturaleza del foco en la lengua española está asociada con la interfaz que se presenta entre sintaxis y entonación. Es decir, a pesar de que en una lengua como el español el patrón acentual no marcado es asignado al grupo melódico del extremo derecho de la oración ${ }^{29}$, el orden de palabras en una estructura

${ }^{28}$ Marina Fernández Lagunilla y Elena de Miguel, "Relaciones entre el léxico y la sintaxis: adverbios de foco y delimitadores aspectuales", Verba, 26 (1999), 97-128; Julia HoRVARTH, Focus in the theory of grammar and the syntax of Hungarian, Foris, Dordrecht, 1986, y R. VAN VALIN, An introduction to syntax, Cambridge University Press, Cambridge, 2001.

${ }^{29}$ M.L. Zubizarreta, art. cit.; Laura Domínguez, "The effects of phonological cues on the syntax of focus constructions in Spanish”, en 
focal está afectado, tanto por los elementos prosódicos como por las restricciones sintácticas. A este respecto, Domínguez menciona, por ejemplo, que el foco en español se correlaciona con la prominencia del acento, generando una alternativa para la regla de acentuación nuclear ${ }^{30}$, la cual está profundamente relacionada con el orden lineal de los constituyentes. De esta manera, el análisis de una oración a partir del nivel de la frase fonológica requiere de un orden que favorezca la medición correcta de la realización del foco y sus efectos en el concierto de constituyentes. Dicho de otro modo, el orden de constituyentes está motivado por factores fonológicos, en particular, el alineamiento del acento (stress) con los entornos iniciales y finales de mayor prominencia tonal ( high pitch) ${ }^{31}$. De este modo, es posible que se prefiera un orden particular de un determinado constituyente o palabra, en un contexto comunicativo, para que el tonema de una frase fonológica esté alineado; por ejemplo, con el sujeto en el orden OVS o VADS, como en (27):

(27) a. Las piedras las quitó el jardinero OVS.

b. Está trabajando en el Ley el tío de Rosa VADS.

En estas dos construcciones, se evidencia un cambio en el orden de constituyentes, desde un orden no marcado (SVO) a uno marcado (OVS), como en (27a) y (VADS), como en (27b); esto con el fin de que el sujeto se ubique en una posición en la que se puede alinear con la mayor prominencia entonativa ${ }^{32}$.

Romance languages and Linguistic. Selected papers from 'going romance', eds. R. Bok-Bennema, B. Hollebrandse, B. Kampers-Manhe \& P. Sleeman, J. Benjamins, Amsterdam, 2002, pp. 69-71 y R. GutiérREz-Bravo, art. cit.

${ }^{30}$ NoAm Chomsky \& Halle Morris, The sound pattern of English, Harper, New York, 1969; Guglielmo Cinque, "A null theory of phrase and compound stress", LI, 24 (1993), 239-267 y TANyA REINHART, "Interfase economy: Focus and markedness”, Studia Gramatica, 1995, núm. 40, 147-169, entre otros.

${ }^{31}$ LARS FANT, Estructura informativa en español. Estudio sintáctico y entonativo, Acta Universitatis Upsaliensis, Uppsala, 1984; Juan Manuel Sosa, La entonación del español. Su estructura fónica, variabilidad y dialectología, Cátedra, Madrid, 1999; Тнгмотну FAce, "Local intonational marking of Spanish contrastive focus", Probus, 14 (2002), 71-92, e Ignacio Hualde, "El modelo métrico y autosegmental", en Teorías la entonación, coord. P. Prieto, Ariel, Barcelona, 2003, pp. 155-184.

${ }^{32}$ H. Contreras, op. cit.; M.L. Zubizarreta, art. cit.; L. Domínguez, art. cit., y R. GutiérRez-Bravo, art. cit., entre otros. 
La manera indicada y fiable para mostrar la interfaz, que podría presentarse entre prosodia y distribución del focalizador ser en el ordenamiento de las partes de la oración, es por medio de un análisis instrumental de naturaleza fonética. Los análisis espectrográficos de todos los contextos en que aparece ser podrían involucrar una secuencia bitonemática en donde aparezca un tono alto en la frontera del grupo melódico o frase melódica, el cual debería encontrarse en la parte final de la oración. Al parecer, este procedimiento es sencillo, pero requiere de una serie de mediciones milimétricas precisas y meticulosas que en este trabajo no realizo.

Aunque por el momento no dispongo de evidencia empírica que corrobore que hay correlación entre la prominencia entonativa y la estructura de foco en las construcciones con ser focalizador, puedo mostrar algunos hechos lingüísticos que sugieren que estas construcciones pueden seguir este patrón. Como se mencionó arriba, se focalizan todos los constituyentes solamente si se encuentran en posición posverbal, como en (28):

(28) a. Una vez llevaba [era [un vidrio panorámico de un carro]].

b. Nosotros nos vinimos fue para el río.

c. Esto se dañó [fue [por ahí]].

d. La policía lo cogió [fue [al vendedor de fruta]].

En las construcciones de (28) se focalizan todos los constituyentes en posición posverbal y se esperaría, si hay correlación entre el marcador de foco ser y la entonación, que los tonemas de la frase melódica de estas oraciones presenten una estructura tonemática alta. De encontrarse esta correlación, se apoyaría la idea de que la posición final de la lengua española es métricamente fuerte, esto es, el español es un lengua de acento entonativo, lo que significa que la prominencia más rica está expresada fonéticamente a través de la combinación de la frecuencia fundamental (F0), la intensidad o la duración ${ }^{33}$. Ahora, si bien existe la probabilidad de encontrar esta correlación, igualmente no se debe descartar lo contrario, lo cual no debería sorprender, ya

${ }^{33}$ H. Contreras, "Sentencial stress. Word order and the notion of subject in Spanish", en The melody of languages intonations and prosody, eds. L.R. Waugh y C.H. Van Schooneveld, University Park Press, Baltimore, 1980; Mary Beckman et al., "Intonation across Spanish, in the tone and break indices framework", Probus, 14 (2002), 9-36; T. FACE, art. cit., e I. HuAlde, art. cit. 
que hay lenguas en el mundo como el toura que dispone de una compleja marcación de foco que no está correlacionada con la entonación ${ }^{34}$. Además, considero que si bien esta variedad del español ya dispone de un marcador de foco in situ, la parte de la oración focalizada puede o no correlacionarse con un patrón entonativo prominente; es decir, si existe un marcador de foco explícito que a su vez tiene correlación con el orden de las partes de la oración, no debe existir, necesariamente, una correlación adicional con la prominencia entonativa.

El posible desprendimiento de la correlación entre las construcciones con ser focalizador y la entonación se podría presentar en las oraciones en las que ser focaliza dos constituyentes diferentes, como en (29a), en las que ser aparece en las escindidas, como en (29b), o en las que ser focalizador se duplica en el mismo constituyente, como en $(29 c)$ :

(29) a. Yo decía [es [que yo estoy demandando [es [un servicio]]]].

$b$. Es ella que lo hace [es por el bien mío].

c. El agua estaba [era [en vez de estar, fría]], estaba [era [que hervía]].

Los problemas que se tendrían que resolver para dar cuenta de si existe o no correlación entre prominencia entonativa y construcciones con focalizador ser son varios: en primer lugar, sería necesario determinar el fraseo melódico, es decir, si estas construcciones presentan o no un único y constante fraseo melódico; en cualquiera de los casos se debe tipificar la estructura tonemática de cada construcción. En segundo lugar, las escindidas han sido señaladas como construcciones que, por excelencia, emplean mecanismos lingüísticos para expresar el foco, entonces: ¿será posible pensar que aquí hay una doble marcación de foco?, o ¿estas construcciones presentan una estructura focal entonativa particular que absorbe los mecanismos focales sintácticos y el marcador ser?

\section{Conclusión}

Las construcciones con serfocalizador son diferentes a las escindidas. Si bien estas dos comparten el hecho de que los rasgos

${ }^{34}$ R. VAN VALIN, art. cit. 
de número, persona y tiempo de serson ininterpretables, en las escindidas mantiene el estatus de un verbo copulativo, mientras que en las de ser focalizador es un marcador de foco. El comportamiento morfológico de ser como marcador de foco es similar a los clíticos focalizadores de otras lenguas, como el quechua, que tienen una estructura focal específica, es decir, con restricciones y dominios específicos.

El focalizador ser tiene dominio focal sobre diferentes constituyentes. Dentro del foco estrecho pueden ser focalizados el sujeto, el objeto directo, el objeto indirecto, los adjuntos y los adverbios, y dentro del foco amplio sólo puede ser focalizado un predicado. En este dominio no puede focalizarse la oración completa porque ser no puede aparecer en posición inicial absoluta.

El clítico focalizador ser presenta una estructura focal rígida, es decir, únicamente se podrán focalizar los constituyentes que se encuentren en posición posverbal. Esta restricción provoca que se presente una correlación entre el orden de constituyentes y el focalizador ser, pues para que el sujeto, que dentro del orden no marcado se ubica en una posición canónica preverbal, sea focalizado, tiene que moverse a una posición posverbal, para situarse en el dominio focal de ser. Por otra parte, de la misma manera que ocurre en otras lenguas, como el sesotho y el toura, el constituyente focalizado con ser se coloca en el extremo derecho de la oración (foco no marcado), pero igualmente se focalizan constituyentes que se encuentran al interior de la construcción (foco marcado).

La correlación que posiblemente se encuentre entre entonación y focalizador ser es aún un tanto problemática, ya que si bien hay contextos comunicativos en los que el constituyente focalizado se encuentra en el extremo derecho de la oración -y en este caso habría una simetría entre la prominencia entonativa y el constituyente focalizado-, también hay casos en los que la parte focalizada se aleja del núcleo final entonativo, en donde es posible que esta simetría no exista. Finalmente, sería muy ilustrador si se buscara el papel que desempeña la entonación en las construcciones que ofrecen doble marcador de foco.

Armando Mora-Bustos

Escuela Nacional de Antropología e Historia 
